\title{
Biofumigación y solarización como estrategias de manejo de nematodos en tomate (Solanum lycopersicum)
}

Biofumigation and solarization as strategies of management of nematode in tomato (Solanum lycopersicum)

Biofumigação e solarização como estratégias para o manejo de nematóides em tomate (Solanum lycopersicum)

\section{Segundo Curay}

se.curay@uta.edu.ec

https://orcid.org/0000-0002-8559-9627

Universidad Técnica de Ambato, Ambato-Ecuador

\section{Marco Pérez-Salinas}

mo.perez@uta.edu.ec

https://orcid.org/0000-0002-4186-1590

Universidad Técnica de Ambato, Ambato-Ecuador

\author{
José Mangui \\ je.mangui@uta.edu.ec \\ https://orcid.org/0000-0001-7928-826X \\ Universidad Técnica de Ambato, Ambato-Ecuador

\section{Norma Telenchana} \\ normatelenchana@hotmail.com \\ https://orcid.org/0000-0001-7417-0828 \\ Universidad Técnica de Ambato, Ambato-Ecuador
}

\section{Carlos Vásquez \\ ca.vasquez@uta.edu.ec \\ https://orcid.org/0000-0002-8214-3632 \\ Universidad Técnica de Ambato, Ambato-Ecuador}

\section{RESUMEN}

El control de los nematodos fitoparásitos se ha basado principalmente en el uso de productos químicos, los cuales cada vez presentan menor eficiencia, por lo que se requiere buscar estrategias ecológicamente sustentables. Se realizaron dos ensayos para evaluar el efecto de la biofumigacion y solarización sobre las poblaciones de nematodos en el cultivo de tomate (Solanum lycopersicum) bajo condiciones de invernadero. En el primer ensayo se estudió el efecto solo y combinado de la biofumigación con la solarización en el valle Quillan, Cantón Píllaro. En el segundo ensayo se evaluó el efecto del estado fenológico de la mostaza de abisinia en el control de nematodos en suelo en la Parroquia La Matriz del Cantón Píllaro. Se observó menor efecto en el control de Meloidogyne sp. (apenas 25\%), mientras que en Xiphinema sp. provocó control total. No se observó efecto de la edad del material vegetal y el tipo de aplicación sobre el número de nematodos, sin embargo, si se observó efecto por el tiempo de acción de los extractos. Basados en los resultados, el uso de brasicáceas en combinación con solarización podría ser una alternativa viable para el manejo de poblaciones de nematodos en cultivos de tomate.

Palabras clave: Biofumigación; Brassicaceae; Estrategias sustentables; Solarización
ABSTRACT

The control of phytoparasitic nematodes has been mainly based on the use of chemical products, which are increasingly less efficient, so it is necessary to seek ecologically sustainable strategies. Two tests were carried out to evaluate the effect of biofumigation and solarization on nematode populations in a tomato crop (Solanum lycopersicum) under greenhouse conditions. In the first trial, the single and combined effect of biofumigation with solarization was studied in Quillan valley, Municipality of Pillaro. In the second trial, the effect of the phenological stage of the Abyssinian mustard on the control of soil-nematodes in Parroquia La Matriz, municipality of Pillaro was evaluated. Less effect was observed in the control of Meloidogyne (barely $25 \%$ ), while in Xiphinema it caused total control. No effect was observed of the age of the plant material and the type of application on the number of nematodes; however, an effect was observed by the time of action of the extracts. Based on the results, the use of Brassicaceae in combination with solarization could be a viable alternative for the management of nematode populations in tomato crops.

Key words: Biofumigation; Brassicaceae; Sustainable strategies; Solarization

\section{RESUMO}

O controle dos nematóides fitoparasitários tem sido feito principalmente através do uso de produtos químicos, cada vez menos eficientes, e por isso é necessário buscar estratégias ecologicamente mais sustentáveis. Dois testes foram realizados para avaliar o efeito da biofumigação e solarização sobre as populações de nematóides na cultura de tomate (Solanum lycopersicum) sob casa de vegetação. No primeiro ensaio, o efeito único e combinado da biofumigação com solarização foi estudado no vale Quillan, Cantón Píllaro. No segundo experimento, avaliou-se o efeito do estado fenológico da mostardada-abissínia no controle de nematóides do solo na Parroquia La Matriz, Cantón Píllaro. Menor efeito foi observado no controle de Meloidogyne sp. (apenas 25\%), enquanto em Xiphinema sp. observou-se controle total. Não foi observado efeito da idade do material vegetal e do tipo de aplicação sobre o número de nematóides, porém, foi observado efeito pelo tempo de ação dos extratos. Com base nos resultados obtidos, o uso de brassicáceas em combinação com a solarização pode ser uma alternativa viável para o manejo de populações de nematóides na cultura do tomate.

Palavras-chave:

Biofumigação; Brassicaceae; Estratégias sustentáveis; Solarização 


\section{INTRODUCCIÓN}

A nivel mundial, los cultivos hortícolas son seriamente amenazados por el ataque de los nematodos agalladores pertenecientes al género Meloidogyne Göldi, principalmente en los países tropicales y subtropicales donde se encuentran ampliamente distribuidos (1). Entre las estrategias de control de las poblaciones de las especies de nematodos se incluye el uso de bromuro de metilo (BM), sin embargo, dados los efectos negativos de este producto, se requiere la búsqueda de alternativas con eficiencia similar pero con menor impacto al ambiente, más económica y socialmente viable $(2,3)$. Así, tanto la biosolarización como la biofumigación han probado ser estrategias ecológicas viables para el manejo de estos patógenos. En tal sentido, Phani et al. (4) señalaron el efecto positivo de la biosolarización sobre el control de Meloidogyne sp. en cultivos de tomate, así como de las malezas. Sin embargo, la eficacia de control sobre las poblaciones de nematodos no es concluyente y esta depende de los niveles poblacionales, de las características del suelo y de la evolución de las temperaturas del suelo a lo largo del ciclo de cultivo (5).

Con relación a la biofumigación, esta se refiere a la liberación de compuestos volátiles a partir de la descomposición de material vegetal o animal y que tienen actividad pesticida (6). Entre las familias botánicas, las especies del género Brassica (Brassicaceae) presentan altos contenidos de glucosinolatos que son capaces de liberar productos volátiles tóxicos (thiocianatos e isothiocianatos) durante la biodegradación (7). Estudios previos han sugerido que estos compuestos tienen un efecto sobre los nematodos habitantes del suelo, sin embargo, estos resultados aún no parecen ser concluyentes. Por un lado, Lord et al. (8) demostraron que la reducción de la viabilidad de quistes de Globodera pallida presentes en el suelo cubierto con plástico y tratado con los restos vegetales de Brassica juncea se debía a los altos contenidos de 2-propenil glucosinolato, sugiriendo que este podría sustituir el uso de fumigantes sintéticos. De manera similar, Aires et al. (9) demostraron que existe una asociación entre el tipo y concentración de glucosinolatos y la disminución de nuevos quistes de Globodera rostochiensis. Contrariamente, McLeod y Steel (10) encontraron que la supresión de Meloidogyne javanica no parecía estar relacionada con el contenidos de los glucosinolatos. Sin embargo, esta falta de relación pudiera ser debida a la forma de incorporación del material vegetal pudo haber dificultado la hidrólisis de los glucosinolatos y consecuentemente la producción de isotiaciantos (8).

En Ecuador, el tomate riñón se cultiva principalmente en las provincias de Imbabura, Cotopaxi, Chimborazo, Tungurahua, Azuay y Loja, sin embargo, la producción ha mostrado una amplia variación desde el 2004 hasta el 2015 debido a la disminución tanto de la superficie cosechada y el rendimiento (11). Esta baja en el rendimiento se debe, entre otras causas, al ataque de plagas, incluyendo los nematodos agalladores. En tal sentido, en el presente estudio se evaluó el efecto 
de la biofumigacion y solarización sobre las poblaciones de nematodos en un cultivo de tomate (Solanum lycopersicum) bajo condiciones de invernadero.

\section{MATERIALES Y MÉTODOS}

Fueron realizados dos ensayos para evaluar el efecto de la biofumigación y solarización sobre las poblaciones de nematodos en un cultivo de tomate (Solanum lycopersicum) bajo condiciones de invernadero. En el primer ensayo se estudió el efecto solo y combinado de la biofumigación y solarización sobre las poblaciones de nematodos en el valle Quillan, Cantón Píllaro con temperatura media anual $13,5^{\circ} \mathrm{C}$, precipitación media anual $1468 \mathrm{~mm}$ y humedad relativa $86,6 \%$ (datos obtenidos de la Estación meteorológica del Colegio "Jorge Álvarez", Cantón Píllaro). El ensayo fue realizado bajo condiciones de invernadero, el cual había sido cultivado con tomate riñón durante aproximadamente 4 años con fertirrigación y sin incorporación de materia orgánica.

Al inicio del ensayo fueron preparadas 10 camas $(0,8 \times 12 \mathrm{~m})$ a las cuales se incorporó restos vegetales de mostaza de abisinia (Brassica carinata) recolectada a los 60 días antes de la floración, previamente triturado hasta obtener un tamaño entre 2 y $3 \mathrm{~cm}$. Este material fue aplicado a razón de 5 (D1) y 10 $\mathrm{kg} / \mathrm{m}^{2}$ (D2) siguiendo a Chango (2015) y se usó una parcela sin ningún tipo de aplicación como control.Seguidamente, elsuelo fuehumedecido hasta capacidad de campo por medio de sistema de riego a goteo y posteriormente las camas fueron recubiertas con polietileno
(50 y $100 \mu \mathrm{m}$ ) para la solarización. Cada tres días fue registrada la temperatura del suelo en horas de la tarde mediante un geotermómetro insertado en distintos lugares de cada tratamiento a una profundidad de 10 $\mathrm{cm}$. Las poblaciones iniciales y finales de los diferentes géneros de nematodos asociados al cultivo fueron estimadas mediante la toma de muestras compuestas de $500 \mathrm{~g}$ de suelo ( $25 \mathrm{sub}$ muestras) a $15 \mathrm{~cm}$ de profundidad y en zig-zag. Las muestreas fueron llevadas al Laboratorio de Nematología del Departamento de Sanidad Vegetal en la Estación Experimental Santa Catalina, Instituto Nacional de Investigaciones Agropecuarias (INIAP). Una vez en el laboratorio, las muestras fueron procesadas mediante el método del "elutriador de Oostenbrink" y "filtro de algodón" en $100 \mathrm{~g}$ de suelo siguiendo la metodología de (12). Posteriormente se realizó la identificación del género tanto de hábitos fitopatógenos y saprofitos y adicionalmente la determinación del número poblacional.

El ensayo fue conducido en un diseño en parcelas divididas, siendo la parcela principal el grosor del polietileno usado para la solarización y las subparcelas representadas por la cantidad de material vegetal (B. carinata) aplicada. Cada tratamiento fue repetido cinco veces.

En el segundo ensayo se evaluó el efecto del estado fenológico de la mostaza de abisinia y el tiempo de acción del material vegetal sobre el control de nematodos en suelo. Este ensayo se llevó a cabo en la Parroquia La Matriz del Cantón Píllaro. 
Previo a la incorporación de la mostaza, el material vegetal de 60 (antes de la floración) y 110 días (después de la floración) fue triturado en trozos de 2-3 cm de largo y posteriormente aplicado al suelo. En ambos casos, se tomaron muestras de suelo al inicio y final del ensayo para determinar la cantidad de nematodos en 100 g desueloy procesadas mediante el método de extracción del embudo de Baermann (12) y la cuantificación fue hecha mediante el método de la cuadrícula usando placas de conteo bajo aumento de un microscopio.

Análisis estadístico: Los datos poblacionales fueron sometidos a análisis de varianza y posteriormente sometidos a prueba de medias de Tukey $(p<0,001)$ usando el programa estadístico Statistix versión 10.

\section{RESULTADOS Y DISCUSIÓN}

\section{Nematodos asociados a tomate riñón}

Fueron identificados especímenes pertenecientes a los géneros Meloidogyne, Xiphinema, Criconemoides, Trichodorus y Tylenchorrhynchus. Del total de nematodos identificados, los géneros Meloidogyne y Xiphinema fueron los más abundantes al inicio del ensayo, mientras que Criconemoides, Trichodorus y Tylenchorrhynchus aparecieron solo al final del ensayo. Estudios previos han reportado diferentes géneros $\mathrm{y} / \mathrm{o}$ especies de nematodos asociados con este cultivo. En Ghana, Osei et al. (13) reportaron ocho especies que incluyeron Helicotylenchus spp., Hoplolaimus indicus, Pratylenchus brachyurus, Rotylenculus reniformis, Scutellonema spp.,
Tylenchulus spp., Xiphinema elongatum y Meloidogyne incognita, siendo esta última la de mayor distribución y con una abundancia relativa del $36,8 \%$, mientras que Hoplolaimus indicus fue la menos abundante. De manera similar, Giesbrecht-Harder y Aquino-Jara (14) encontraron a Meloidogyne sp., Criconemoides sp., Trichodorus sp y Tylenchorhynchus sp., entre los principales nematodos fitoparasíticos asociados con el cultivo de tomate, además de Helicotylenchus sp., Dorylaimus sp., Tylenchus sp. y Ditylenchus sp.

\section{Efecto de la mostaza de abisinia y solarización sobre las poblaciones de nematodos}

Los resultados del primer ensayo demostraron que hubo efecto de los tratamientos (biofumigación y solarización) sobre las poblaciones de Meloidogyne y Xiphinema $(\mathrm{p}<0,01 ; \mathrm{F}=70,0 ; \mathrm{gl}=4$ ) (Figura 1a, b). Se observó que el uso de $5 \mathrm{~kg} / \mathrm{m}^{2}$ de mostaza de abisinia logró reducir en 80,0 y $71,25 \%$ las poblaciones de Meloidogyne, independientemente del grosor del plástico usado para la solarización, mientras que con el uso de $10 \mathrm{~kg} / \mathrm{m}^{2}$ solo se logró entre 43,75 y $50 \%$ de control de estos nematodos. En el caso de Xiphinema, todos los tratamientos provocaron control total, debido probablemente a una mayor susceptibilidad a los biofumigante usados. Con relación al resto de los géneros de nematodos encontrados no fue posible establecer el nivel de control puesto que estos no habían aparecido al inicio del ensayo. 
En general se considera que las especies de Meloidogyne representan una seria amenaza para el cultivo de tomate riñón a nivel mundial, debido a que este presenta un ciclo de vida corto y una alta tasa de reproducción por lo que pueden incrementar rápidamente sus poblaciones y consecuentemente provocar daño económico $(13,15)$. El resto de los nematodos encontrados, aunque pueden actuar como fitoparásitos de hortalizas y otros cultivos, aun su impacto económico no ha sido determinado (13).

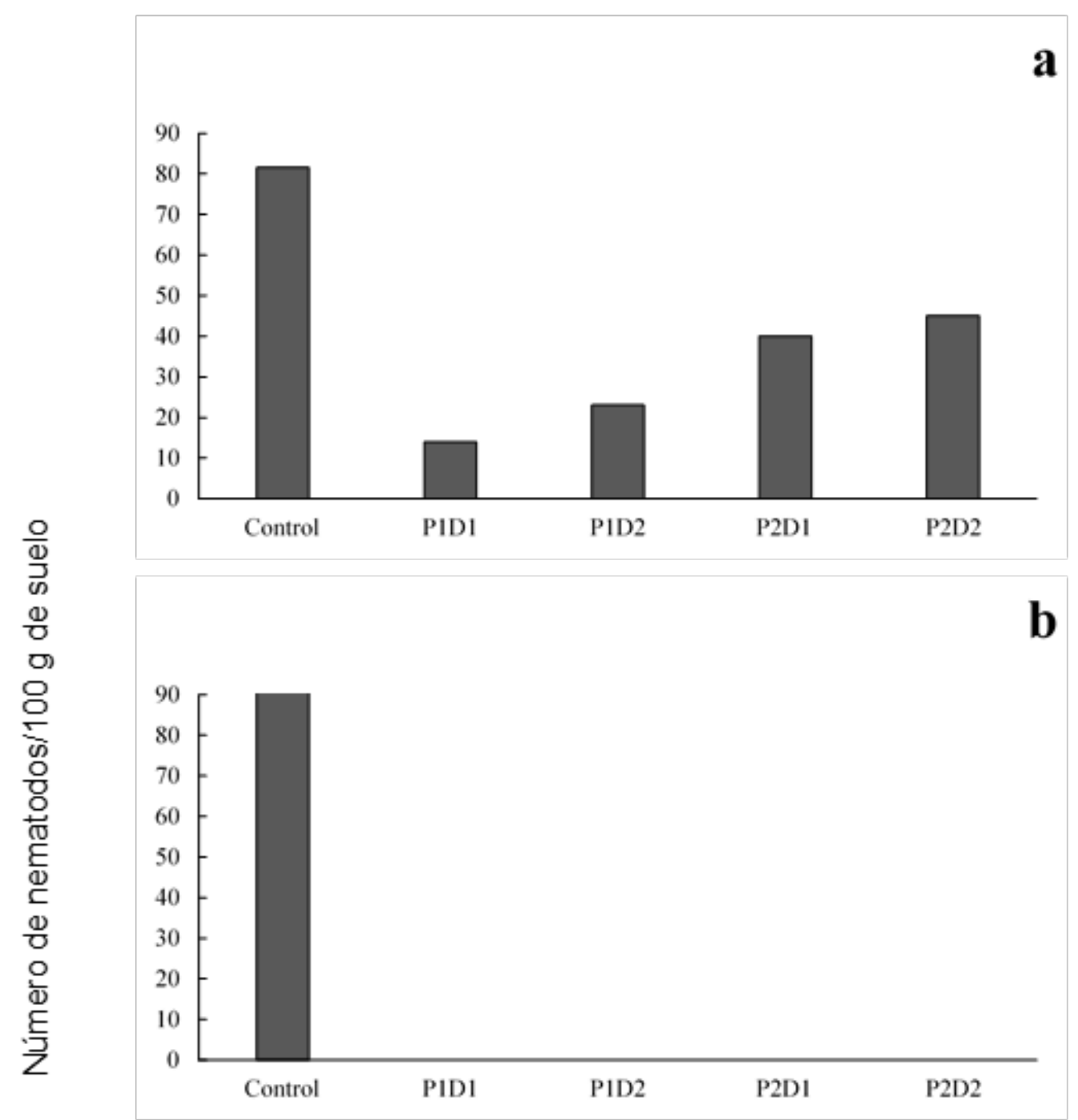

Figura 1. Variación en el número de nematodos de los géneros Meloidogyne (a) y Xiphinema (b) por efecto de la biofumigación con mostaza de abisinia y solarización $\left(\mathrm{P} 1=5 \mathrm{~kg} / \mathrm{m}^{2} ; \mathrm{P} 2=10 \mathrm{~kg} / \mathrm{m}^{2} ; \mathrm{D} 1=\right.$ plástico de $50 \mu \mathrm{m}$; $\mathrm{D} 2=$ plástico de $100 \mu \mathrm{m})$. 
De manera similar, Pokharel (16) afirmó que Meloidogyne fue controlado más eficientemente solo con el uso de biofumigantes, mientras que el uso combinado con solarización o gallinaza no se reflejó en mejores niveles de control. Contrariamente, estos autores notaron que la disminución de las poblaciones de Pratylenchus fue más evidente cuando se aplicó conjunta de biofumigación con solarización.

En el segundo ensayo, el número de nematodos/100 g de suelo varió tanto por la edad como del tiempo de acción del material vegetal (Figura 2). A los 60 días después de aplicado el material vegetal, el número de
Meloidogyne se redujo $63,12 \%$ en las parcelas donde se aplicó restos de mostaza de abisinia colectada antes de la floración, mientras que esta reducción fue de apenas 38,56\% cuando se usó restos de mostaza de abisinia colectados en floración. De manera interesante se observó que los restos de mostaza de abisinia colectada requirieron mayor tiempo para ejercer mayor efecto de control, puesto que se alcanzó un 80,07 \% de disminución de la población de nematodos a los 110 días después de la aplicación, en comparación con un 68,13\% alcanzado con el material colectado antes de la floración.

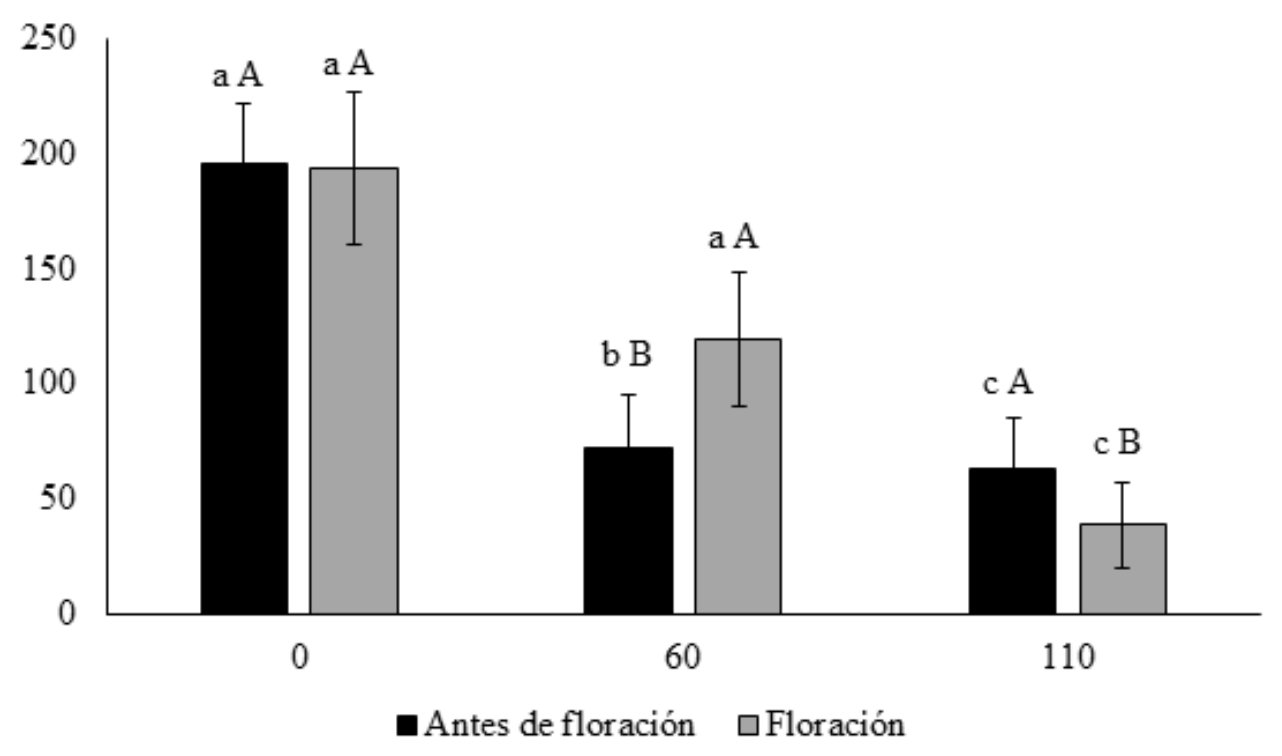

Figura 2. Número de nematodos en $100 \mathrm{~g}$ de suelos cultivados de tomate por efecto de la aplicación de restos vegetales de B. carinata colectados en dos estados fenológicos. 
Barras con la misma letra minúscula no mostró diferencias significativas por efecto del tiempo de aplicación (0, 60 y 110 días); barras con la misma letra mayúscula no mostraron diferencias significativas por efecto del estado fenológico del biofumigante. Prueba de media según Tukey $(p<0,001)$.

Estudios previos han demostrado el efecto biofumigante de las especies de Brassicaceae sobre especies de nematodos. López-Elías et al. (17) encontraron que tanto la biofumigación con Brassica oleraceae var. italica provocó la disminución del número de nematodos en un $34 \%$ en plantaciones de sandía. Sin embargo, el porcentaje de control puede variar de acuerdo con las especies usadas. En tal sentido, Lord et al. (8), demostraron que los cultivares de Raphanus sativus cv. Weedcheck (rábano), Nasturtium officinale (berro), y Brassica juncea cv. Nemfix (mostaza hindú), fueron particularmente más potentes puesto produjeron entre 97 y $89 \%$ de inhibición en comparación con aquellas variedades bajas en glucosinolatos, las cuales provocaron un efecto bajo sobre M. incognita. Aparte de la especie, la forma de partición del material vegetal también influye en la actividad biofumigante. Así, la mayor producción de glucosinolato es asegurada cuando el material vegetal es usado antes de llegar a plena floración y aplicado previamente picado pues esto último asegura la hidrólisis que provocará la liberación de altas concentraciones de isotiocianatos volátiles con actividad biocida (18). Aparte del efecto biocida, los biofumigantes favorecen la multiplicación de microorganismos del suelo que compiten con los patógenos favoreciendo la disminución de la incidencia de enfermedades en plantas y por otra parte, esta práctica permite incorporar fertilizantes verdes en el suelo (19).

\section{CONCLUSIONES}

Basados en los resultados, la edad del material vegetal y el tipo de aplicación no mostraron tener ningún efecto sobre el número de nematodos en el suelo, sin embargo, se observó que mientras más tiempo permanecía este material en el suelo provocaba mejor control de los nematodos. Adicionalmente, se observó que el uso la mostaza de abisinia en combinación con solarización mostró un efecto positivo en el control de nematodos del suelo, en tal sentido esta práctica podría ser una alternativa viable para el manejo de poblaciones de nematodos en cultivos de tomate en la región de la Sierra ecuatoriana.

\section{REFERENCIAS BIBLIOGRÁFICAS}

1. Gómez L, González E, Enrique R, Hernández MA, Rodríguez MG. Uso de biofumingación para el manejo de Meloidogyne spp., en la producción protegida de hortalizas. Rev Protección Veg. 2010;25(2):119-23

2. Ruzo LO. Physical, chemical and environmental properties of selected chemical alternatives for the pre-plant use of methyl bromide as soil fumigant. Pest Manag Sci. 2006;62(2):99-113

3. Duniway JM. Status of chemical alternatives to methyl bromide for pre-plant fumigation of soil. Phytopathology. 2002;92(12):1337-43

4. Phani V, Khan MR, Dutta TK. Plant-parasitic nematodes as a potential threat to protected agriculture: Current status and management 
options. Crop Prot [Internet]. 2021;144:105573. Available from: https://doi.org/10.1016/j. cropro.2021.105573

5. BelloA, López-PérezJ.,García-ÁlvarezA,Arcos SC, Ros C, Guerrero MM, et al. Biofumigación con solarización para el control de nematodos en cultivo de pimiento. In: Lacasa A, Guerrero MM, Oncina M, Mora JA, editors. Desinfección de suelos en invernaderos de pimiento. Murcia: Publicaciones de la Consejería de Agricultura, Agua y Medio Ambiente; 2004. p. 129-208

6. Brennan RJB, Glaze-Corcoran S, Wick R, Hashemi M. Biofumigation: An alternative strategy for the control of plant parasitic nematodes. J Integr Agric [Internet]. 2020;19(7):1680-90. Available from: http:// dx.doi.org/10.1016/S2095-3119(19)62817-0

7. Dutta TK, Khan MR, Phani V. Plant-parasitic nematode management via biofumigation using brassica and non-brassica plants: Current status and future prospects. Curr Plant Biol [Internet]. 2019;17(December 2018):1732. Available from: https://doi.org/10.1016/j. cpb.2019.02.001

8. Lord JS, Lazzeri L, Atkinson HJ, Urwin PE. Biofumigation for control of pale potato cyst nematodes: Activity of brassica leaf extracts and green manures on globodera pallida in vitro and in soil. J Agric Food Chem. 2011;59(14):7882-90

9. Aires A, Carvalho R, da Barbosa MC, Rosa E. Suppressing potato cyst nematode, Globodera rostochiensis, with extracts of Brassicacea plants. Am J Potato Res. 2009;86(4):327-33

10. McLeod RW, Steel CC. Effects of brassicaleaf green manures and crops on activity and reproduction of Meloidogyne javanica. Nematology. 1999;1(6):613-24

11. MAGAP. Boletín situacional de tomate riñón [Internet]. 2015. Available from: http://sinagap. agricultura.gob.ec/phocadownloadpap/ cultivo/2016/boletin_situacional_tomate_ rinon_2015.pdf

12. Nijs D. Nematode extraction PM 7/119. EPPO Bull. 2013;43(3):471-95

13. Osei K, Osei MK, Mochiah MB, Lamptey JNL, Berchie JN. Plant parasitic nematodes associated with tomato in Ghana. Nematol Mediterr. 2012;40(1):33-7

14. Giesbrecht Harder SB, Aquino Jara AS. Control alternativo de nematodos en el cultivo de tomate (Lycopersicon esculentum Mill.) en condiciones de invernadero. Investig Agrar. 2009;11(1):29-35

15. Hirunsalee A, Barker KR, Beute MK. Infection, reproduction potential, and root galling by root-knot nematode species and concomitant populations on peanut and tobacco. J Nematol. 1995;27(2):172-7

16. Pokharel R. Soil Solarization, an alternative to soil fumigants [Internet]. Vol. 2, Crop Series/ Soils. 2012. Available from: http://extension. colostate.edu/docs/pubs/crops/00505.pdf

17. López-Elías J, Guerrero-Ruíz JC, HuezLópez MA, Jiménez-León J, Ruiz-Mendoza JJ. Biofumigación y solarización del suelo en la producción de sandía (Citrullus lunatus (Thumb.) Matsum. \& Nakai). Eur Sci J. 2014;10(3):121-31

18. dos Santos CA, Abboud AC de S, Do Carmo MGF. Biofumigation with species of the brassicaceae family: A review. Cienc Rural. 2021;51(1):1-17

19. Devi G. Biofumigation: A Potential Aspect for Suppression of Plant-Parasitic Nematodes. Int J Environ Agric Biotechnol. 2018;3(4):128591 